\title{
Development and validation of a Food Choices Score for use in weight-loss interventions
}

\author{
Sara J. Grafenauer ${ }^{1 *}$, Linda C. Tapsell ${ }^{1}$, Eleanor J. Beck ${ }^{1}$ and Marijka J. Batterham ${ }^{2}$ \\ ${ }^{1}$ Smart Food Centre, School of Health Sciences, University of Wollongong, Northfields Avenue, \\ Wollongong, 2522 NSW, Australia \\ ${ }^{2}$ School of Mathematics and Applied Statistics, University of Wollongong, Northfields Avenue, \\ Wollongong 2522 NSW, Australia \\ (Submitted 16 May 2013 - Final revision received 9 December 2013 - Accepted 3 January 2014 - First published online 6 February 2014)
}

\section{Abstract}

Weight loss results from an energy deficit, although the quality of food choices making up the diet may also be important. The aim of the present study was to develop and validate a diet quality tool based on food categories to monitor dietary change in clinical weight-loss settings. The Food Choices Score (FCS) was based on seventeen food categories, each scoring up to five points, totalling 85 . In addition to content validity, the tool was validated using (1) two energy-deficit diet models (6500 and $7400 \mathrm{~kJ}$ ) assuring nutrient and food-group targets and (2) dietary data from two weight-loss trials ( $n$ 189). First, the diet models confirmed that an optimal score of 85 was achievable. Second, change in scores was compared with weight loss achieved at 3 months. The trial data produced a mean FCS of $42 \cdot 6$ (SD 8.6), increasing to $49 \cdot 1$ (SD 7.6) by 3 months. Participants who lost weight achieved a higher FCS at 3 months than those who did not $(P=0.027)$, and there was an even greater improvement in the FCS $(P=0.024)$ in participants losing $\geq 5 \%$ body weight than in those losing $<5 \%$. A greater change in the FCS $(\Delta \geq 7)$ resulted in a greater change in BMI $(P=0.044)$, and score change was correlated with weight change $(P=0.023)$. Participants with the highest scores $(\geq 56 v . \leq 44 / 85)$ consumed more fruit $(P<0 \cdot 001)$ and low-fat dairy foods $(P=0.004)$, less fatty meat $(P<0 \cdot 001)$, non-whole-grain cereals $(P<0 \cdot 001)$, non-core foods and drinks (NCFD) ( $P<0 \cdot 001)$, less energy $(P=0.018)$, less dietary fat $(P<0.001)$ and more dietary fibre $(P=0.013)$. Weight loss was $35.5 \%$ less likely to be achieved with every increase in the serves of NCFD $(P=0.004)$ in the study sample. The FCS is a valid tool for assessing diet quality in clinical weight-loss settings.

\section{Key words: Weight reduction: Food intakes: Nutrition assessment}

Weight loss results from an energy deficit, although the quality of food choices making up the diet may also be important ${ }^{(1,2)}$. In examining this idea, an observational cohort study of 4-year weight change has found that weight gain was most strongly associated with intakes of meat (processed and unprocessed), potatoes, potato chips and sugar-sweetened beverages and inversely associated with free vegetables, fruit, whole-grain foods, nuts and yogurt ${ }^{(3)}$. This study has provided some suggestion of specific foods of interest in weight loss. The recognition that we 'eat foods, not nutrients' sounds simplistic $^{(4)}$, but it signals a paradigm shift from focusing on nutrient composition to food composition of the whole diet, embracing the concept of food synergy ${ }^{(5-11)}$. Encompassing this concept, diet quality tools have emerged in epidemiological research, moving the focus from single nutrients to a whole-diet-based perspective in relation to disease ${ }^{(12)}$, and research now focusing entirely on the macronutrient proportions of the diet may be of limited value ${ }^{(13)}$. The definition of diet quality used in constructing a tool depends on the attributes selected by the researcher $^{(14)}$. In a review, Reul ${ }^{(15)}$ found no official definition of dietary quality, yet the concept of quality of energy is gaining support at the research level ${ }^{(16)}$. Historically, dietary quality referred to nutrient adequacy, and implied that the diet met requirements for essential nutrients within energy requirements ${ }^{(15)}$. In the management of chronic conditions such as obesity $^{(17)}$ and the metabolic syndrome ${ }^{(18)}$, a diet of highquality food choices is essential and forms an integral layer of dietary advice. However, high diet quality may be more difficult to achieve within an energy restriction, and interventions tend to report energy- and nutrient-level changes, but not changes in diet quality ${ }^{(1)}$.

A diet quality tool is a predefined measure based on food groups and/or nutrients, or dietary guidelines and creates a single quantifiable rank or score by subject ${ }^{(19)}$. Several reviews

Abbreviation: FCS, Food Choices Score.

*Corresponding author: S. J. Grafenauer, fax +6124221 484, email sara@nourishnutrition.com.au 
of diet quality tools have been published ${ }^{(14,17,19-24)}$, defining important considerations in the methodological process of designing such tools and the differences between tools. The most recent review by Wirt \& Collins ${ }^{(17)}$ examined twenty-five indices of diet quality or diet variety that used a range of measures from nutrients to food servings or food groups. This review noted many methodological weaknesses in the existing tools, but concluded that higher diet quality was inversely related to all-cause mortality with a moderate protective effect. The 'moderate' effect size was generalised since the predictive capacity of most indices was reportedly in a similar range; that is, a $17-42 \%$ reduction in all-cause mortality, a $18-53 \%$ reduction in CVD mortality, a $14-28 \%$ reduction in the risk of CVD, a $13-30 \%$ reduction in cancer mortality and a $7-35 \%$ reduction in all-cancer risk ${ }^{(17)}$.

A number of diet quality tools are available for dietary pattern research, yet many are based on dietary guidelines including the Diet Quality Index ${ }^{(25)}$, the Healthy Eating Index ${ }^{(26)}$ and the Dietary Guideline Index ${ }^{(27)}$, and only some have been validated for certain populations ${ }^{(21,27,28)}$. Few studies have assessed the effect of diet quality in terms of weight change in an intervention setting $^{(1,19)}$. The published studies have tended to use an existing tool that includes both foods and nutrients ${ }^{(2,29)}$ or a tool based on dietary guidelines ${ }^{(1,30)}$, or a tool that does not include all of the possible foods and drinks consumed ${ }^{(31,32)}$. None of these studies has used a tool specifically designed for clinical weight loss, and this setting may require a more specific tool to correctly depict dietary change. The aim of the present study was to develop and validate a diet quality tool based on food categories to monitor dietary change in clinical weight-loss interventions.

\section{Methods}

Reference data for the analyses described herein were obtained from diet history records from two clinical weight-loss trials and included the participants completing 3 months ( $n$ 195). This sample has been described previously ${ }^{(33)}$. Each trial was approved by the University of Wollongong Human Research Ethics Committee and registered with Australian New Zealand Clinical Trials Register Network (12608000425392 and 12610000784011). Both trials were based on individualised energy restriction $(80 \%$ of BMR $\times$ physical activity level 1.25 using the Mifflin St Jeor equation ${ }^{(34)}$ ) and focused on achieving a prescribed intake of core foods with high dietary quality. Diet history data reflective of a weekly pattern of intake were collected by Accredited Practising Dietitians. Before the interview, participants completed a $4 \mathrm{~d}$ food record that assisted with recall of types and amounts of foods consumed. A checklist of specific foods including their frequency of consumption was also used for items that may have been omitted from the history records. Household measures and food models were used as a prompt for serve size. All food records were analysed using a computerised food and nutrient database, FoodWorks $^{\mathrm{TM}}$ Professional (version 6, 2009; Xyris). Underreporters were excluded using the Goldberg cut-off limits $(0 \cdot 76-1 \cdot 24)^{(35,36)}$, reducing the sample size for the analysis presented herein ( $n$ 189).
The Food Choices Score (FCS) was developed based on seventeen food categories, and the scope of foods from within each food category has been adopted from previous research ${ }^{(33)}$. Each food item reported in the diet history interviews was entered into the computerised food and nutrient database, categorised according to the described groups and then analysed in $\mathrm{g}$ and $\mathrm{kJ}$. The number of serves of each food category was calculated in grams (except for alcoholic beverages and the non-core foods and drinks category where the number of serves was calculated in kJ). Serve sizes were adapted from two ready reckoners ${ }^{(37,38)}$ and have been used in previously published research ${ }^{(33)}$.

The 3-month diet history data were used to guide the development of the scoring scale as these data represented the improved, prescribed diet. To define the scoring scale for each food category, the number of serves (per $d$ ) for each food category was ranked from lowest to highest consumption that was examined graphically, noting the range (maximum and minimum number) of serves consumed. The highest score was adjusted as required in line with the recommended serves for each food group ${ }^{(39,40)}$. Reverse scoring, i.e. lower scores for highest consumption, was applied to food categories for which consumption limits (associated with negative health outcomes) have been documented in the literature, e.g. fatty meats have been linked with chronic disease $^{(41-43)}$. A U-shaped scoring scheme was used for food groups for which benefits exist with limited consumption but negative consequences with excess intake ${ }^{(21)}$, e.g. alcohol. Alcohol consumption within the recommended limits ${ }^{(44)}$ may provide some benefit to health and is not associated with weight gain $^{(45)}$, but heavier consumption over time is associated with weight gain ${ }^{(46)}$ and other negative health outcomes $^{(47-49)}$.

A scoring scale in serves per $\mathrm{d}$ with scores ranging from 0 to 5 aligned with increments for each food category was identified to achieve a maximum FCS of 85 (Table 1). The highest score applied to each food category reflected the optimal range of intake based on the described considerations. Scores were applied to the serve-based data of each trial participant ( $n$ 189) at baseline and 3 months using equations in Microsoft Excel (2010) to ensure accuracy of the composite score.

Content validity involved a qualitative check of possible methodological weaknesses according to the latest review of diet quality scores ${ }^{(17,21)}$. This check addressed key issues relating to the content of the diet quality score as described by Waijers et al. ${ }^{(21)}$, including the choice of the index components and the assignment of food items to food categories (Table 2), for example distinguishing between whole grains and refined grains ${ }^{(17,50)}$, assessing dairy foods and dairy food alternatives by fat content rather than Ca content, and providing separate categories for fruit and vegetables, and fish and seafood ${ }^{(21)}$. Food preparation was also taken into account in accordance with our previously published work $^{(33)}$. For example, plain boiled or steamed starchy vegetables were assigned to the starchy vegetables category, while fried potatoes (or chips) were assigned to the non-core foods and drinks category. Similarly, fried meats such as schnitzel were assigned to the fatty meats category. 


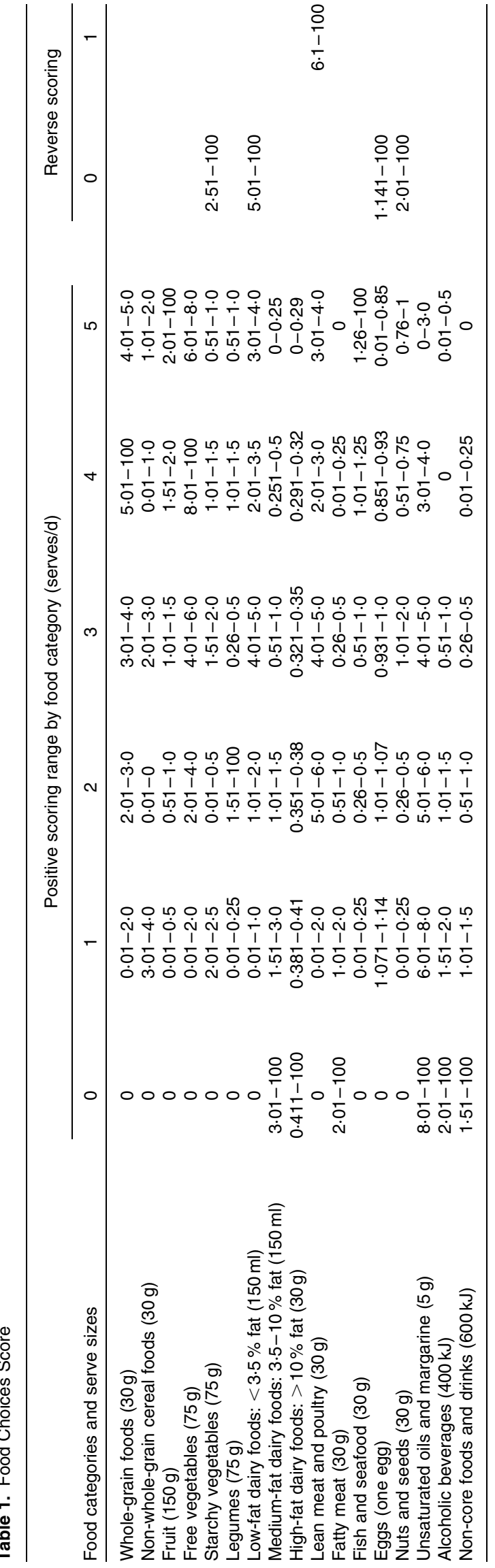

Construct validity evaluated quantitatively how well the scoring system measured what it was supposed to measure. This was assessed in two ways. First, two theoretical energy-deficit diet models were constructed (based on 6500 and $7400 \mathrm{~kJ}$ ), representing the highest diet quality score of 85 . The upper and lower boundary limits for energy intake were based on the mean reported energy intake of females (6031 (SD $1100) \mathrm{kJ}$ ) and males $(7274$ (SD 1752) kJ) at the 3-month time point, and on the energy intake range of the diet prescriptions for female $(5000-7500 \mathrm{~kJ})$ and male $(6500-9000 \mathrm{~kJ})$ participants. Both the mean (reported) and prescribed energy intakes were taken into consideration in order to accommodate both men and women within the highest score, and this score was validated through the modelling of food categories ${ }^{(21)}$ (Table 3). The tool was specifically designed to prevent higher diet quality being the result of purely increasing energy intake, rather higher diet quality was based on specific food choices and specifically reflected lower energy intake resulting in weight loss (Table 3 ). The nutrient value of the associated range of serves by food category was tested using data from the FoodWorks ${ }^{\mathrm{TM}}$ Professional software system (version 6, 2009; Xyris) in comparison to food guide recommendations (in serves) in use for the healthy population ${ }^{(40)}$, and Nutrient Reference Values (Suggested Dietary Targets and Estimated Average Requirements) ${ }^{(39)}$ (Table 3). Second, using the trial data, change in the FCS was compared with weight loss achieved at 3 months in the trials. Thus, internal validity was demonstrated by comparing diet quality scores in idealised diets using the diet models, nutrient values and recommended number of serves from the national guidelines ${ }^{(51)}$, while external validity was demonstrated by comparing the highest $(\geq 70 \%)$ and the lowest $(\leq 60 \%)$ scores in relation to food categories, energy intake and nutrients consumed.

\section{Statistical analyses}

The compatibility of the two combined trial databases in terms of age of participants, BMI, reported percentage of macronutrients consumed and a $\chi^{2}$ test tested for sex differences between groups at baseline has been established and reported previously ${ }^{(33)}$. Independent-samples $t$ tests were used to evaluate differences in the FCS at baseline and 3 months between sexes to ensure there was no sex effect.

To test the validity of the FCS, (1) the maximum FCS was calculated using the idealised diet model and (2) the FCS values were used to estimate the relationship between the score, food categories and weight loss. The mean (and standard deviation or $95 \% \mathrm{CI}$ ) and range values of the FCS and the change in score were calculated for the total sample at each time point. The values for participants who lost weight were compared with the values for those who did not lose weight, for those that lost greater than (and less than) 5\% body weight and for those scoring greater than the mean change in score using independent-samples $t$ tests. Overall, three score bands were formed to distinguish between the participants scoring below $60 \%$ of the total score $(\leq 44 / 85)$ and those scoring above $70 \%$ of the total score $(\geq 56 / 80)$. Food category data (at baseline and 3 months), energy intake and nutrients 
Table 2. Validation plan outlining content and construct validity considerations ${ }^{(17,21,23,57)}$

\begin{tabular}{|c|c|}
\hline Content validity & Construct validity \\
\hline $\begin{array}{l}\text { Food category considerations } \\
\text { Other published indices } \\
\text { Choice of food categories, e.g. fish } \\
\text { separate from meat }{ }^{(21)} \text { and dairy foods categorised } \\
\text { by fat content rather than Ca content }{ }^{(21)} \\
\text { Need to reflect the diet, the } \\
\text { extremes of consumption and recommended consumption } \\
\text { Subcategories based on food type } \\
\text { Food preparation effects, e.g. higher-fat cooking } \\
\text { methods } \\
\text { Food processing effects, e.g. refined and } \\
\text { whole grains }{ }^{(17,50)} \\
\text { Nutrients } \\
\text { Nutrients not included - separate analysis performed } \\
\text { Diet quality score } \\
\text { Overall diet quality determined by the tool rather } \\
\text { than as a subjective measure }\end{array}$ & $\begin{array}{l}\text { Diet models considering } \\
\text { Energy } \\
\text { Food recommendations } \\
\text { Nutrient reference values } \\
\text { Scoring } \\
\text { Reverse scoring and U-shaped scoring } \\
\text { (for meat and alcohol) or a combination } \\
\text { of these }(21,58) \\
\text { More than two scoring points per } \\
\text { category } \\
\text { Scores achieved by participants } \\
\text { Baseline } \\
3 \text { months } \\
\text { Change in score compared with weight } \\
\text { loss }\end{array}$ \\
\hline
\end{tabular}

consumed were analysed using a one-way ANOVA with post hoc Bonferroni correction. Normality of the data was determined using the Shapiro-Wilk test, and then comparison between weight change and score change was made using Pearson's correlation coefficient. Logistic regression was used to determine whether weight loss was predicted by increasing or decreasing intakes of particular food categories in the total sample. All statistical analyses were performed using IBM SPSS Statistics (version 19.0.0; IBM Corporation).

\section{Results}

The maximum diet quality score of 85 was shown to meet food guide recommendations (in serves) and Nutrient Reference Values (Table 3). The Goldberg cut-off limits excluded six participants due to under-reporting at baseline, reducing the sample size ( $n$ 189) in the present analysis. At baseline, no differences were found between men and women in terms of reported energy intake or nutrients consumed (carbohydrate, protein, fat or dietary fibre). At 3 months, men reported a significantly reduced energy intake compared with women $(-3935$ (SD 3017) $v$. -2715 (SD 1832) kJ; $P=0.010$ ). At baseline, the mean FCS was 42.6 (SD 8.6) (range 19-61/85) and at 3 months, it was 49.1 (SD 7.6) (range 28-68/85). There was no difference found in mean FCS between the participants who lost weight $(n$ 177) and those who did not (42.7 (SD 8.7) v. 41.1 (SD 6.5); $P=0.531$ ) at baseline, whereas a difference was found between the participants who lost weight and those who did not (49.4 (SD 7.4) v. $44.4(\mathrm{SD} 10 \cdot 0) ; P=0.027)$ at 3 months.

At baseline, a difference was found in score for the participants who lost more than $5 \%$ body weight ( $n$ 100/189) (40.9 ( $\operatorname{sD} 8.5) v .44 .5$ (SD 8.3); $P=0.003$ ), in favour of the group who lost less weight at 3 months. At 3 months, there was no significant difference in score (49.1 (SD 7.1) v. $49 \cdot 2$ (SD 8.2); $P=0.967)$, although there was a difference in score change $(\Delta=8.3$ (SD 10.9) v. 4.6 (SD 11.1); $P=0.024)$, in favour of the weight-loss group. For the total sample, the mean change in the FCS was 7 (SD 11). When the score change value was greater than the mean change $(\Delta \geq 7 ; n$ 100) for the total sample, BMI change was greater $(\Delta=-1 \cdot 8($ SD $1 \cdot 1)$ v. -1.5 (SD 1.1); $P=0.044)$.

Participants with the highest scores at 3 months (FCS $\geq 56 / 85$ ) had a greater score change value, significantly higher than those with the lowest scores $(\Delta=14.4$ (SD 8.4) $v .-2 \cdot 7$ (SD 10.2); $P<0.001)$. The score change between the lowest and highest score bands was also reflected in an improvement in diet quality (in eight out of seventeen food categories), exemplified by a greater consumption of fruit $(P<0 \cdot 001)$, more consumption of low-fat dairy foods $(P=0 \cdot 003)$, more consumption of legumes $(P=0.032)$, less consumption of medium-fat dairy foods $(P<0.001)$, less consumption of higher-fat dairy foods $(P=0 \cdot 001)$, less consumption of fatty meat $(P<0 \cdot 001)$, less consumption of non-whole-grain (refined) cereals $(P<0.001)$ and less consumption of non-core foods and drinks $(P<0.001)$. The changes in the dietary pattern resulted in a significant difference in the intakes of energy $(P=0 \cdot 018)$, total dietary fat $(P<0 \cdot 001)$ and dietary fibre $(P=0 \cdot 031)$ (Table 4$)$.

Pearson's correlation coefficient analysis revealed that a mean score change of 6.5 (SD 11.1) points was correlated with a mean weight change of -4.7 (SD 3.0$) \mathrm{kg}$; although this correlation was significant, it was weak $(P=0 \cdot 023 ; 0 \cdot 165)^{(52)}$. Logistic regression analysis using the available sample revealed that for every one serve increase in non-core foods and drinks, the odds of weight loss was 0.645 (reduced by $35.5 \%$; $P=0.004)$, and that with every one serve increase in non-whole-grain (refined) cereals, the odds of weight loss was 0.825 (reduced by $17 \cdot 5 \% ; P=0 \cdot 011$ ). Therefore, increasing consumption of non-core foods and drinks and non-wholegrain cereals was less likely to lead to weight loss. Although increasing fruit consumption was less significant in comparison $(P=0.061)$, weight loss was 1.485 times more likely to be achieved for every one serve increase in consumption.

\section{Discussion}

Analyses using the FCS demonstrated the achievement of a maximum score in an idealised diet, and associations between 
Table 3. Diet model for the highest Food Choices Score rating of 85

\begin{tabular}{|c|c|c|c|c|}
\hline \multirow[b]{2}{*}{ Food categories and serve sizes } & \multicolumn{2}{|c|}{ Proposed serve range } & \multicolumn{2}{|c|}{ Food guide recommendations (healthy population) ${ }^{(40,59)}$} \\
\hline & Lower bound & Upper bound & Minimum & Maximum \\
\hline Whole-grain foods (30 g) & 4 & 5 & 5 & 8 \\
\hline Non-whole-grain cereals $(30 \mathrm{~g}$ ) & 1 & 2 & NR & 2 \\
\hline Fruit $(150 \mathrm{~g})$ & 2 & 2 & 2 & 4 \\
\hline Free vegetables $(75 \mathrm{~g})$ & 6 & 8 & 3 & 6 \\
\hline Starchy vegetables $(75 \mathrm{~g})$ & 0.5 & 1 & 1 & 4 \\
\hline Legumes $(75 \mathrm{~g})$ & 0.5 & 1 & & Unlimited \\
\hline Low-fat dairy foods: $<3.5 \%$ fat $(150 \mathrm{ml})$ & 3 & 4 & & Total dairy: 4 (higher-fat dairy foods $<40 \mathrm{~g}$ ) \\
\hline Medium-fat dairy foods: $3 \cdot 5-10 \%$ fat $(150 \mathrm{ml})$ & 0 & 0.25 & & \\
\hline High-fat dairy foods: $>10 \%$ fat $(30 \mathrm{~g})$ & 0 & 0.29 & & \\
\hline Lean meat and poultry $(30 \mathrm{~g})$ & 3 & 4 & & $<455 \mathrm{~g} /$ week \\
\hline Fatty meat $(30 \mathrm{~g})$ & 0 & 0 & & NR \\
\hline Fish and seafood $(30 \mathrm{~g})$ & 1 & 1.26 & & $20-40 \mathrm{~g}$ \\
\hline Eggs (one egg) & 0.01 & 0.86 & & 6 per week \\
\hline Nuts (and seeds) $(30 \mathrm{~g})$ & 0.7 & 1 & & $60 \mathrm{~g}$ \\
\hline Unsaturated oils and margarine $(5 \mathrm{~g})$ & 3 & 3 & & $7 \mathrm{~g}$ oil; $10 \mathrm{~g}$ margarine \\
\hline Alcoholic beverages $(400 \mathrm{~kJ})$ & 0.5 & 0.5 & & NR \\
\hline \multirow[t]{2}{*}{ Non-core foods and drinks (600 kJ) } & 0 & 0 & & 1 \\
\hline & \multicolumn{2}{|c|}{ Nutrient analysis } & \multicolumn{2}{|c|}{$\begin{array}{l}\text { Suggested dietary targets or estimated } \\
\text { average requirement }{ }^{(39)}\end{array}$} \\
\hline Energy (kJ) & 6499 & 7381 & & \\
\hline Protein $(\mathrm{g})$ & 85 & 93 & & \\
\hline$\%$ & 21 & 21 & 15 & 25 \\
\hline Fat $(\mathrm{g})$ & 50 & 59 & & \\
\hline$\%$ & 27 & 30 & 20 & 35 \\
\hline Saturated fat (g) & $12 \cdot 4$ & 15 & & \\
\hline$\%$ & 7 & 7.5 & - & $<7$ \\
\hline Polyunsaturated fat (g) & $10 \cdot 0$ & $11 \cdot 6$ & - & - \\
\hline Monounsaturated fat (g) & 23.0 & $27 \cdot 5$ & - & - \\
\hline Carbohydrate $(\mathrm{g})$ & 181 & 188 & & \\
\hline$\%$ & 44 & 42 & 45 & 65 \\
\hline Alcohol (g) & 6.5 & 6.5 & - & - \\
\hline Dietary fibre (g) & 34.0 & 34.5 & F 28 & M 38 \\
\hline Vitamin C (mg) & $288 \cdot 0$ & 296.0 & F 190 & M 220 \\
\hline Total folate $(\mu \mathrm{g})$ & $486 \cdot 0$ & 466.0 & 300 & 600 \\
\hline $\mathrm{Ca}(\mathrm{mg})$ & 992.0 & $1096 \cdot 0$ & 840 & 1100 \\
\hline $\mathrm{Fe}(\mathrm{mg})$ & 11.5 & $13 \cdot 0$ & F 8 & M 6 \\
\hline $\mathrm{Zn}(\mathrm{mg})$ & 11.0 & $11 \cdot 3$ & F 6.5 & M 12 \\
\hline
\end{tabular}

$N R$, no recommendation; $F$, female; $M$, male.

better quality food choices and weight loss, in a setting where high-quality foods were advised. The FCS utilised the key suggestions by Waijers et al. ${ }^{(21)}$ in terms of content and met the food group and Nutrient Reference Values in an idealised diet model with the highest score of 85. A higher FCS was consistent with improved diet quality and was associated with increased consumption of fruit, legumes and low-fat dairy foods (closer to the requirements) and decreased consumption of mediumand higher-fat dairy foods, fatty meat, non-whole-grain (refined) cereals and, importantly, non-core foods and drinks. These food-level changes reflect those also noted by Mozaffarian et $a l .{ }^{(3)}$ within an observational cohort described earlier. By segmenting participants based on weight loss, it was apparent that those losing the most body weight (>5\%) increased their score significantly by 3 months. The highest scores were a reflection of the degree of achievement in terms of diet quality and dietary change over time, although the highest possible score was not achieved by any participants in the sample. Thus, we considered the FCS to be valid and reliable in that the highest score was achieved in an idealised diet model, and the identified changes in the consumption of foods using the FCS were consistent with observational studies of foods that were negatively associated with weight loss. These, of course, are qualitative assessments, and we did not provide an exact measure of precision. Logistic regression using the entire sample suggested that certain foods were more likely to be associated with weight loss, and these same food categories were identified by the FCS.

A diet quality tool can fulfil a number of purposes, and, to date, they have been used to support disease predictions, outcome measures and monitoring of foods, food groups, nutrients or combination of these ${ }^{(14,17,21)}$. Many of the tools developed have provided a relative score or an assessment against Dietary Guidelines ${ }^{(21)}$, and have not been tailored for the intervention setting ${ }^{(28)}$. It has been suggested that a diet quality tool would be suited to the diet assessment process ${ }^{(17)}$. Furthermore, there are suggestions in the literature $^{(2)}$ that choosing particular foods such as nuts ${ }^{(53)}$ and whole foods $v$. more processed foods ${ }^{(54)}$ may better support weight maintenance. One of the arguments is that the 
Table 4. Low ( $\leq 60 \%)$, medium and high ( $\geq 70 \%)$ Food Choices Scores (FCS) by food category, energy intake and nutrients consumed at 3 months $(n 189)^{*}$

(Mean values and standard deviations)

\begin{tabular}{|c|c|c|c|c|c|c|c|c|}
\hline \multirow[b]{2}{*}{ Food categories and serve sizes } & \multicolumn{2}{|c|}{$\begin{array}{c}\text { Low scores } \\
(\mathrm{FCS} \leq 44)(n 51)\end{array}$} & \multicolumn{2}{|c|}{$\begin{array}{c}\text { Medium scores } \\
\text { (FCS 45-55) ( } n \text { 95) }\end{array}$} & \multicolumn{2}{|c|}{$\begin{array}{c}\text { High scores } \\
(\mathrm{FCS} \geq 56)(n 43)\end{array}$} & \multicolumn{2}{|c|}{$P$} \\
\hline & Mean & SD & Mean & SD & Mean & SD & $\begin{array}{l}\text { Between } \\
\text { groups }\end{array}$ & $\begin{array}{l}\text { Post hoc } \\
\text { low v. high }\end{array}$ \\
\hline Whole-grain foods (30 g) & $2 \cdot 8$ & 1.9 & $3 \cdot 0$ & 1.8 & $3 \cdot 1$ & 1.4 & 0.690 & 1.000 \\
\hline Non-whole-grain cereals $(30 \mathrm{~g})$ & 3.5 & $3 \cdot 0$ & 1.9 & $1 \cdot 6$ & $1 \cdot 7$ & 1.4 & $<0.001$ & $<0.001$ \\
\hline Fruit $(150 \mathrm{~g})$ & $1 \cdot 2$ & 0.6 & 1.6 & 0.8 & 1.7 & 0.6 & $<0.001$ & $<0.001$ \\
\hline Free vegetables $(75 \mathrm{~g})$ & 4.2 & $2 \cdot 2$ & $5 \cdot 1$ & 1.8 & $5 \cdot 1$ & 1.9 & 0.018 & 0.056 \\
\hline Starchy vegetables $(75 \mathrm{~g})$ & $1 \cdot 0$ & 0.9 & 0.9 & 0.6 & 0.9 & 0.5 & 0.754 & 1.000 \\
\hline Legumes $(75 \mathrm{~g})$ & 0.3 & 0.5 & 0.5 & 0.6 & 0.7 & 0.7 & 0.005 & 0.004 \\
\hline Low-fat dairy foods: $<3.5 \%$ fat $(150 \mathrm{ml})$ & $2 \cdot 1$ & 1.6 & $2 \cdot 7$ & 1.4 & 3.0 & $1 \cdot 2$ & 0.005 & 0.004 \\
\hline Medium-fat dairy foods: $3 \cdot 5-10 \%$ fat $(150 \mathrm{ml})$ & 0.6 & 1.2 & 0.1 & 0.3 & 0.03 & 0.1 & $<0.001$ & $<0.001$ \\
\hline High-fat dairy foods: $>10 \%$ fat $(30 \mathrm{~g})$ & 0.4 & 0.4 & 0.2 & 0.5 & 0.1 & $0 \cdot 1$ & 0.005 & 0.003 \\
\hline Lean meat and poultry ( $30 \mathrm{~g}$ ) & $3 \cdot 2$ & 1.7 & $3 \cdot 4$ & $2 \cdot 7$ & $3 \cdot 0$ & 1.4 & 0.547 & 1.000 \\
\hline Fatty meat $(30 \mathrm{~g})$ & 0.9 & 0.9 & 0.4 & 0.6 & 0.3 & 0.5 & $<0.001$ & $<0.001$ \\
\hline Fish and seafood $(30 \mathrm{~g})$ & $1 \cdot 1$ & 0.9 & 1.5 & 1.2 & 1.7 & $1 \cdot 2$ & 0.059 & 0.091 \\
\hline Eggs (one egg) & 0.4 & 0.5 & 0.3 & 0.2 & 0.4 & 0.2 & 0.302 & 0.805 \\
\hline Nuts (and seeds) ( $30 \mathrm{~g}$ ) & 0.4 & 0.4 & 0.3 & 0.4 & 0.3 & 0.3 & 0.326 & 0.493 \\
\hline Unsaturated oils and margarine $(5 \mathrm{~g})$ & $2 \cdot 4$ & $3 \cdot 7$ & $2 \cdot 1$ & 2.5 & 1.4 & 1.4 & $0 \cdot 174$ & 0.204 \\
\hline Alcoholic beverages (400 kJ) & 0.6 & 1.0 & 0.6 & 0.8 & 0.5 & 0.7 & 0.702 & 1.000 \\
\hline Non-core foods and drinks (600 kJ) & 1.8 & $1 \cdot 3$ & $1 \cdot 2$ & 0.8 & 0.6 & 0.5 & $<0.001$ & $<0.001$ \\
\hline \multicolumn{9}{|l|}{ Nutrients } \\
\hline Energy (kJ) & 6833 & 1484 & 6206 & 1383 & 6041 & 1213 & 0.010 & 0.018 \\
\hline Protein $(\mathrm{g})$ & $86 \cdot 3$ & $15 \cdot 1$ & 83.8 & 21.4 & $84 \cdot 4$ & $18 \cdot 8$ & 0.755 & 1.000 \\
\hline Fat $(\mathrm{g})$ & $52 \cdot 2$ & $15 \cdot 8$ & $42 \cdot 8$ & 14.5 & 38.9 & $13 \cdot 3$ & $<0.001$ & $<0.001$ \\
\hline Carbohydrate (g) & $181 \cdot 0$ & $42 \cdot 4$ & $165 \cdot 4$ & 39.8 & $164 \cdot 3$ & $30 \cdot 6$ & 0.044 & 0.114 \\
\hline Dietary fibre $(\mathrm{g})$ & $25 \cdot 7$ & $6 \cdot 8$ & $27 \cdot 1$ & $5 \cdot 7$ & 29.5 & $6 \cdot 4$ & 0.016 & 0.013 \\
\hline
\end{tabular}

*One-way ANOVA with post hoc Bonferroni correction.

metabolisable energy of the unprocessed food is less than the estimated available energy (reflected in food composition tables), whereas food processing may increase the availability of energy. So a measure of diet quality is helpful. Specifically, the present analysis highlighted a decreased consumption of non-whole-grain (refined) cereals and non-core foods and drinks in weight loss, as confirmed by logistic regression analysis further supporting the conclusions using the FCS.

Designing an index of diet quality is highly complex. Many tools have been validated in populations; however, they may have been incorrectly applied in different contexts ${ }^{(21,27,28)}$. There are many forms of dietary scores, and there are calls in the literature to be clear regarding the intention of the score $^{(17,21)}$. If an index is based on dietary guidelines, it provides a relative measure against that standard, or if the index is designed with specific culturally based dietary elements, it should really only be applied to that specific population. The FCS was developed to measure diet quality specifically in a weight-loss context in which the dietary advice focused on high-quality foods. Many arbitrary choices have been made in designing past tools and applying scores ${ }^{(21)}$. The advantage of the FCS is that it was developed using contextsensitive dietary data and pre-tested with theoretical diet models. This differentiated the FCS from tools appropriate for use at the population level. To develop this clinical research tool, there was a need to define sensible, datadriven cut-off points for each food category so as not to overemphasise a single food category variable. It is not plausible that all index components contribute equally to the total score or to the same health outcome ${ }^{(21)}$, and this is an issue for some existing tools. The score range for each food category was then validated within the theoretical diet models to ensure that the highest score could accommodate current nutrient targets and food recommendations without exceeding the energy range for males and females. Consequently, an alignment with energy, nutrient and food category targets was considered to be of importance in designing the FCS. While energy restriction is pivotal to weight loss, this can compromise nutrient intake or nutrient status ${ }^{(55)}$. In recent research of dietary patterns at the baseline stage of a clinical trial, we found that weight loss was more easily achieved when poor-quality diets were improved ${ }^{(33)}$. This lead us to consider the concept of a diet quality score and how this might change over time in the trial. The emphasis on diet quality in a weight-loss context recognises the interrelationships between foods and food components, and considers the relationship between the dietary pattern and overall health. Importantly, the FCS was able to capture as much detail on all foods and drinks consumed in the diet as possible, and points to particular foods and drinks as possible targets for the weight-loss setting.

Waijers et al. ${ }^{(21)}$ suggested that a diet quality tool includes a measure of two macronutrients as an assessment of overall dietary balance; however, a check of nutrients can be easily 
conducted as an additional analysis without needing to be incorporated into the tool itself. We were able to demonstrate relevant nutrient changes alongside the food category changes. Rather than including a subjective score for overall diet quality as part of the tool, the overall assessment of diet quality was determined by the tool itself and the final $\mathrm{FCS}^{(21)}$. The present study demonstrates that the change in the FCS discerned differences in diet quality since the total score was able to distinguish between the participants with improved consumption habits and those with less consumption habits, and the FCS at 3 months differentiated those with a greater change in BMI and an overall change in score was correlated with weight loss, even though all subjects were prescribed the same energy deficit. Application of the FCS demonstrated that participants can achieve weight loss, although improving diet quality, meeting nutrient requirements and reducing intakes of non-core foods and drinks appear to be an important step in achieving this outcome.

The FCS diet index tool was based on data from a small population of overweight to obese subjects ( $n$ 189). An important consideration is the interpretation of the score and understanding the limitations of the tool and the score. In the present study, no participants achieved greater than $80 \%$ of the possible FCS, and it is recommended that the tool be tested with a group within the healthy weight range to further assess the validity of the tool. Food classification was central to the way in which the FCS was developed, and there are questions as to the classification of foods: first, in relation to nutritional homogeneity within the categories ${ }^{(15)}$ and, second, food classification is influenced by how foods are viewed culturally ${ }^{(15)}$. As with all dietary assessment methods, the FCS is context-sensitive and may need modification for other clinical settings. The food categories and the serve size of each category used in the FCS have been utilised in previously published research ${ }^{(33)}$, and the food categories selected reflected the current recommendations concerning foods and food groups in relation to weight loss ${ }^{(15,21)}$. Ensuring that the tool captures the current emphasis on diet-disease relationships represents a limitation, and the FCS would need to be adjusted as new evidence about specific foods is established. Finally, all dietary studies must deal with the issue of misreporting of dietary data, particularly among overweight participants ${ }^{(56)}$. In the present study, under-reporters were removed using the Goldberg cut-off limits ${ }^{(35,36)}$.

\section{Conclusion}

The FCS proved to be valid when applied to an idealised diet model, and the highest FCS represented higher diet quality discerning the differences in energy and nutrient intakes. Furthermore, weight loss was related to a greater improvement (change) in the FCS, suggesting that examination of the changing pattern of foods consumed during weight loss is informative and complements the change in macronutrient intakes. The ability to deliver specific food advice in the clinical setting is pivotal to changed dietary behaviour, and these findings suggest that particular foods and beverages may be able to be targeted in weight-loss advice. The FCS was specifically designed to align with energy-, nutrient- and food-based recommendations, and, together, the analysis of the food categories, energy intake, nutrients consumed, body weight loss and change in BMI helps validate the FCS. The highest scores using the FCS indicated improved diet quality as a result of dietary change and represent increased reported consumption of positive, core food choices and decreased consumption of non-core food and drinks and non-whole-grain cereal choices, giving specific direction for advice in practice. The FCS proved to be valid for assessing diet quality in clinical weight-loss settings, producing maximum scores in the optimised diet models and demonstrating expected changes in food choice patterns under supervised weight-loss conditions

\section{Acknowledgements}

The authors thank Nathan Larkin, Research Fellow from the Faculty of Engineering for his assistance with data management.

The present study was supported by an Australian Postgraduate Award, and the trials from which the data were made available for this analysis were funded by the National Health and Medical Research Council and Horticulture Australia Limited (ACTRN 12608000425392 and 12610000784011). The funding bodies had no role in the design, analysis or writing of this article.

The authors' contributions are as follows: S. J. G. was responsible for conceptualising the study, data analysis and the initial and final drafts of the manuscript; E. J. B. and L. C. T. wrote the manuscript; M. J. B. provided guidance regarding the statistical analyses. All authors read and approved the final manuscript.

The authors declare that they have no conflicts of interest.

\section{References}

1. Webber KH \& Lee E (2011) The diet quality of adult women participating in a behavioural weight-loss programme. J Hum Nutr Diet 24, 360-369.

2. Nazare J-A, Smith J, Borel A-L, et al. (2013) Changes in both global diet quality and physical activity level synergistically reduce visceral adiposity in men with features of metabolic syndrome. J Nutr 143, 1074-1083.

3. Mozaffarian D, Hao T, Rimm EB, et al. (2011) Changes in diet and lifestyle and long-term weight gain in women and men. $N$ Engl J Med 364, 2392-2404.

4. Jacques PF \& Tucker KL (2001) Editorial: are dietary patterns useful for understanding the role of diet in chronic disease. Am J Clin Nutr 73, 1-2.

5. Patterson RE, Haines PS \& Popkin BM (1994) Diet quality index: capturing a multi-dimensional behavior. $J$ Am Diet Assoc 94, 57-64.

6. Kant AK, Schatzkin A, Graubard BI, et al. (2000) A prospective study of diet quality and mortality in women. JAMA $\mathbf{2 8 3}$, 2109-2115.

7. Messina M, Lampe JW, Birt DF, et al. (2001) Reductionism and the narrowing nutrition perspective: time for reevaluation and emphasis on food synergy. J Am Diet Assoc 101, 1416-1419.

8. Hu FB (2002) Dietary pattern analysis: a new direction in nutritional epidemiology. Curr Opin Lipidol 13, 3-9. 
9. Jacobs DR \& Steffen LM (2003) Nutrients, food and dietary patterns as exposures in research: a framework for food synergy. Am J Clin Nutr 78, 508S-513S.

10. Jacobs DR Jr, Gross MD \& Tapsell LC (2009) Food synergy: an operational concept for understanding nutrition. Am J Clin Nutr 89, 1S-6S.

11. Jacobs DRJ, Tapsell LC \& Temple NJ (2011) Food synergy: the key to balancing the nutrition research effort. Public Health Rev 33, 507-529.

12. Freeland-Graves JH \& Nitzke S (2013) Position of the academy of nutrition and dietetics: total diet approach to healthy eating. $J$ Acad Nutr Diet 113, 307-317.

13. Fogelholm M, Anderssen S, Gunnarsdottir I, et al. (2012) Dietary macronutrients and food consumption as determinants of long-term weight change in adult populations: a systematic literature review. Food Nutr Res 56, 1-45.

14. Kant AK (1996) Indexes of overall diet quality: a review. J Am Diet Assoc 96, 785-791.

15. Reul MT (2003) Operationalizing dietary diversity: a review of measurement issues and research priorities. J Nutr 133, 3911S-3926S

16. Ebbeling CB, Swain JF, Feldman HA, et al. (2012) Effects of dietary composition on energy expenditure during weightloss maintenance. JAMA 307, 2627-2634.

17. Wirt A \& Collins CE (2009) Diet quality - what is it and does it matter? Public Health Nutr 12, 1-19.

18. Eckel RH, Grundy SM \& Zimmet PZ (2005) The metabolic syndrome. Lancet 365, 1415-1428.

19. Togo P, Osler M, Sorensen TI, et al. (2001) Food intake patterns and body mass index in observational studies. Int J Obes Relat Metab Disord 25, 1741-1751.

20. Kant AK (2004) Dietary patterns and health outcomes. J Am Diet Assoc 104, 615-635.

21. Waijers PMCM, Feskens EJM \& Ocke MC (2007) A critical review of predefined diet quality scores. Br J Nutr 97, 219-231.

22. Arvaniti F \& Panagiotakos DB (2008) Healthy indexes in public health practice and research: a review. Crit Rev Food Sci Nutr 48, 317-327.

23. Fransen HP \& Ocké MC (2008) Indices of diet quality. Curr Opin Clin Nutr Metab Care 11, 559-565.

24. Kourlaba G \& Panagiotakos GB (2009) Dietary quality indices and human health: a review. Maturitas 62, 1-8.

25. Haines PS, Siega-Riz AM \& Popkin BM (1999) The Diet Quality Index revised: a measurement instrument for populations. J Am Diet Assoc 99, 697-704.

26. Kennedy ET, Ohls J, Carlson S, et al. (1995) The Healthy Eating Index: design and applications. J Am Diet Assoc 95, $1103-1108$

27. McNaughton SA, Ball K, Crawford D, et al. (2008) An index of diet and eating patterns is a valid measure of diet quality in an Australian population. J Nutr 138, 86-92.

28. Mainvil LA, Horwath CC, McKenzie JE, et al. (2011) Validation of brief instruments to measure adult fruit and vegetable consumption. Appetite 56, 111-117.

29. Valente EA, Sheehy ME, Avila JJ, et al. (2011) The effect of the addition of resistance training to a dietary education intervention on apolipoproteins and diet quality in overweight and obese older adults. Clin Interv Aging 6, 235-241.

30. Wiltheiss GA, Lovelady CA, West DG, et al. (2013) Diet quality and weight change among overweight and obese postpartum women enrolled in a behavioral intervention program. J Acad Nutr Diet 113, 54-62.

31. Cottell KE, Dorfman LR, Straight CR, et al. (2011) The effects of diet education plus light resistance training on coronary heart disease risk factors in community-dwelling older adults. J Nutr Health Aging 15, 762-767.

32. Raynor HA, Looney SM, Steeves EA, et al. (2012) The effects of an energy density prescription on diet quality and weight loss: a pilot randomized controlled trial. J Acad Nutr Diet 112, 1397-1402.

33. Grafenauer SJ, Tapsell LC, Beck EJ, et al. (2013) Baseline dietary patterns are a significant consideration in correcting dietary exposure for weight loss. Eur J Clin Nutr 67, 330-336.

34. Mifflin MD, St Jeor ST, Hill LA, et al. (1990) A new predictive equation for resting energy expenditure in healthy individuals Am J Clin Nutr 51, 241-247.

35. Goldberg GR, Black AE, Jebb SA, et al. (1991) Critical evaluation of energy intake data using fundamental principles of energy physiology: 1 . Derivation of cut-off limits to identify under-recording. Eur J Clin Nutr 45, 569-581.

36. Black AE (2000) Critical evaluation of energy intake using the Goldberg cut-off for energy intake:basal metabolic rate. A practical guide to its calculation, use and limitations. Int J Obes Relat Metab Disord 24, 1119-1130.

37. Curtin University of Technology (2002) Dietitians' Pocket Book. Perth: Curtin University of Technology.

38. Stewart R (editor) (2009) Griffith Handbook of Clinical Nutrition and Dietetics. South Port: Griffith University School of Public Health.

39. Australian Government (2006) Nutrient Reference Values for Australia and New Zealand. Canberra: Department of Health and Ageing, Ministry of Health.

40. National Health and Medical Research Council (2013) $A$ Modelling System to Inform the Revision to the Australian Guide to Healthy Eating: Commonwealth of Australia. Canberra: National Health and Medical Research Council.

41. Micha R, Wallace SK \& Mozaffarian D (2010) Red and processed meat consumption and risk of incident coronary heart disease, stroke, and diabetes mellitus: a systematic review and meta-analysis. Circulation 121, 2271-2283.

42. Pan A, Sun Q, Bernstein AM, et al. (2012) Red meat consumption and mortality results from 2 prospective cohort studies. Arch Intern Med 172, 555-563.

43. Roussell MA, Hill AM, Gaugler TL, et al. (2012) Beef in an Optimal Lean Diet study: effects on lipids, lipoproteins, and apolipoproteins. Am J Clin Nutr 95, 9-16.

44. National Health and Medical Research Council (2009) Australian Guidelines to Reduce Health Risks from Drinking Alcohol. Canberra ACT: National Health and Medical Research Council.

45. Dietary Guidelines Advisory Committee (2010) Report of the Dietary Guidelines Advisory Committee on the Dietary Guidelines for Americans. The Secretary of Agriculture and the Secretary of Health and Human Services. Washington, DC: U.S. Department of Agriculture, Agricultural Research Service.

46. Behall KM, Howe JC \& Anderson RA (2002) Apparent mineral retention is similar in control and hyperinsulinemic men after consumption of high amylose cornstarch. J Nutr 132, 1886-1891.

47. Corrao G, Rubbiati L, Bagnardi V, et al. (2000) Alcohol and coronary heart disease: a meta-analysis. Addiction 95, $1505-1523$.

48. Ridolfo B \& Stevenson C (2001) The Quantification of DrugCaused Mortality and Morbidity in Australia 1998. Canberra: Commonwealth Department of Human Services and Health. http://www.aihw.gov.au/publication-detail/?id= 6442467226

49. Parry C, Patra J \& Rehm J (2011) Alcohol consumption and non-communicable diseases: epidemiology and policy implications. Addiction 106, 1718-1724. 
50. McCullough M, Feskanich D, Rimm E, et al. (2000) Adherence to the Dietary Guidelines for Americans and risk of major chronic disease in men. Am J Clin Nutr 72, 1223-1231.

51. Health and Medical Research Council (2013) Australian Guide to Healthy Eating. Department of Health and Aging. Canberra: National Health and Medical Research Council.

52. Cohen J (1988) Statistical Power Analysis for the Behavioural Sciences, 2nd ed. Hilldale, NJ: Lawrence Erlbaum Associates.

53. Novotny JA, Gebauer SK \& Baer DJ (2012) Discrepancy between the Atwater factor predicted and empirically measured energy values of almonds in human diets. $\mathrm{Am} \mathrm{J}$ Clin Nutr 96, 296-301.

54. Barr SB \& Wright JC (2010) Postprandial energy expenditure in whole-food and processed-food meals: implications for daily energy expenditure. Food Nutr Res 54, 5144.
55. Kant AK (2002) Weight-loss attempts and reporting of foods and nutrients, and biomarkers in a national cohort. Int J Obes 26, 1194-1204.

56. Kuhnle GGC (2012) Nutritional biomarkers for objective dietary assessment. J Sci Food Agric 92, 1145-1149.

57. Wirfält E, Drake I \& Wallström P (2013) What do review papers conclude about food and dietary patterns? Food Nutr Res 57.

58. Di Castelnuovo A, Costanzo S, Bagnardi V, et al. (2006) Alcohol dosing and total mortality in men and women: an updated meta-analysis of 34 prospective studies. Arch Intern Med 166, 2437-2445.

59. NHMRC (2013) The Australian Dietary Guidelines. Canberra: Department of Health and Aging, Commonwealth of Australia. 